\title{
Automatic Extraction of Implanted Electrode Grids
}

\author{
Oskar M. Škrinjar ${ }^{1}$ and James S. Duncan ${ }^{1,2}$ \\ 1 Departments of Electrical Engineering \\ 2 Diagnostic Radiology \\ Yale University, New Haven, CT 06520, USA \\ oskar.skrinjar@yale.edu \\ http://pantheon.yale.edu/ os28/
}

\begin{abstract}
It is common in epilepsy surgery to implant grids and strips of electrodes between the skull and brain or inside the brain, in order to localize functional areas. MR scans are currently used for a variety of image-guided surgical planning tasks, including the localization of the electrode grids. However, the MR scan taken of a patient with implanted electrodes is distorted, and it is difficult to visualize and relate the electrode positions to head and brain structures. For this reason we have developed an automatic algorithm that reliably extracts grids of electrodes from corrupted post-op MR scans. The grid is fitted as a smooth, curved surface through the estimated electrode positions, properly estimating the orientation of the thin disk-shaped electrodes. The extracted grid is then displayed in 3D together with the desired brain structures, coloring the electrodes corresponding to particular functional areas. It is now much easier to visualize and locate the positions of the important functional areas with respect to other brain structures and plan the surgery. This method is currently in clinical use within the Department of Neurosurgery, Yale University and Yale New Haven Hospital.
\end{abstract}

\section{Introduction}

During the course of neurosurgery grids and strips of electrodes are often implanted between the brain and skull or inside the brain and are used to map the brain function. However, in order to effectively use the activation test results, one needs to be able to relate the electrodes to some of the head and brain structures. This is conventionally done by using the post-op MR scans ${ }^{1}$, and looking for the electrodes in the scan. Clinicians find this approach limiting, however, since one can look only at individual 2D image slices, and since one typically sees just a few electrodes in any slice, it is not clear where they are in the grid (e.g. a grid can have 64 electrodes, arranged in an 8 by 8 matrix), and it is difficult to relate them to the brain structures. For this reason we have developed a tool for extracting and visualizing grids of electrodes. The tool extracts the grid as a

\footnotetext{
${ }^{1}$ By post-op MR scan it is meant the MR scan taken after the electrodes have been implanted
} 
whole, not as a set of non-related electrodes, by fitting a smooth, curved surface through the estimated centers of the electrodes. A by-product is the ability to reliably estimate the orientation of the electrodes ${ }^{2}$. Knowing the centers and orientations of the electrodes, and the surface of the grid supporting material ${ }^{3}$, the whole grid is realistically displayed in $3 \mathrm{D}$, together with brain structures of interest. In addition, the electrodes in the grid corresponding to particular functional areas are colored, further helping establish the correspondence between functional areas and brain structures. By using our tool, it is now much easier to reliably locate the functional areas with respect to the other structures, and plan and guide the surgery.

To the best of our knowledge there are no such methods available in the literature. While our method is automatic, there are two other approaches commonly employed: manual selection of electrodes (see [1]), which it is time consuming, or use of a post-op CT scan for electrode detection. Our first results were reported as an abstract (see [2]), while here we present the full method.

\section{Method}

The electrodes we use produce sphere-shaped artifacts of about $10 \mathrm{~mm}$ in diameter in the MR scan (there is a sphere artifact at the position of each electrode). The artifacts appear to be dark, hide the nearby tissue, and in addition they are often mixed with other head structures that are normally dark (e.g. bone, cerebro-spinal fluid - CSF). Moreover, the wires connected to the electrodes produce artifacts, especially where they are joined together (at the place they leave the skull). Fig. 1 shows typical artifacts in post-op scans.

Because of the artifacts and because of the noise one cannot reliably estimate the positions of the electrodes if they are treated separately, and the orientation of electrodes (the orientation of disks) cannot be estimated at all. The idea here in our work is to treat the grid (e.g. an 8 by 8 grid) as a whole. There are two main reasons to do this. First, this includes prior knowledge (the geometry of the grid is known prior to extraction) and second, the errors in estimating the electrode positions tend to cancel out. Our method is based on a combination of nonlinear and predictive filtering subject to differential geometry constraints. The algorithm fits a smooth, curved surface through the estimated electrode positions, and even if an estimated position is significantly off, the geometric constraints push it very close to its true position. As a by-product, once the surface is fitted, one can find the surface normals at positions of the electrodes and use then to properly orient the electrodes (the disks). The orientation of the electrodes is not as important as their position (the aim is to locate the functional areas), but helps to better visualize the grid by making it more realistic.

The constraint that the grid is deformable but not stretchable is used. If one represents the grid surface as a $2 \mathrm{D}$ structure with coordinates $x(s, t), y(s, t)$,

\footnotetext{
${ }^{2}$ Electrodes used at Yale New Haven Hospital are disk-shaped and are $3.8 \mathrm{~mm}$ in diameter and about $.5 \mathrm{~mm}$ in height

3 The grid supporting material is usually transparent rubber
} 


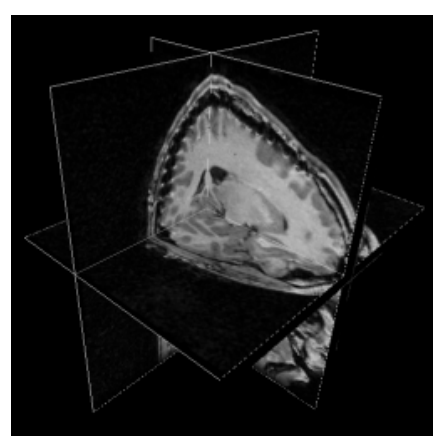

(a)

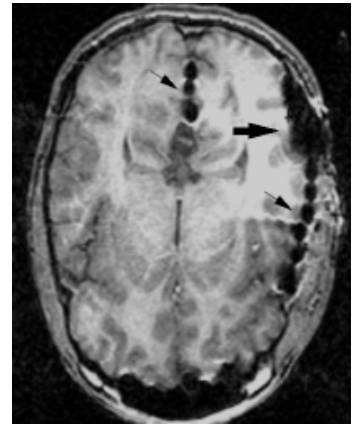

(b)

Fig. 1. Figure (a) shows three orthogonal sections through a post-op MR scan. One can see the artifacts caused by electrodes of an 8 by 8 grid between the top of the brain and the skull. It is not easy to visualize where those electrodes are in the grid and how they are related to brain structures of interest. Figure (b) shows an axial slice of another patient. The artifacts are usually sphere-shaped (small arrows), but sometimes (big arrow) are so dominant that it is very difficult even for the human eye to locate the electrode positions.

$z(s, t)$, then this constraint reduces to the fact that the integral

$$
\int_{s 1}^{s 2} \sqrt{\left(\frac{\partial x}{\partial s}\right)^{2}+\left(\frac{\partial y}{\partial s}\right)^{2}+\left(\frac{\partial z}{\partial s}\right)^{2}} d s
$$

is independent on $t$, and is invariant under the grid deformation. The same holds if $t$ and $s$ exchange positions.

Due to artifacts and noise it is very difficult in one step to reliably fit a smooth grid of a known geometry that satisfies (1). Rather, the geometry and smoothness constraints are enforced through a few steps, each step introducing some portion of the constraints. The three main steps of the method are: nonlinear filtering, predictive filtering and surface regularization and interpolation.

\subsection{Nonlinear Filtering}

The purpose of this step is to find a set of possible electrode centers. Typically, patients are implanted a few grids and strips of electrodes often totaling to more than a hundred electrodes. Since each electrode causes an artifact that is dark, the first idea was to cross correlate a 3D kernel similar to the sphereshaped artifact to the 3D post-op dataset. The maxima of the normalized crosscorrelation would be possible electrode centers. The problem with this is how to set the kernel. While the center of the kernel has to be a dark sphere, the rest of it has to be "white", or some kind of transition to "white". This pattern depends on the structures surrounding particular electrode, and it is not the same for all electrodes. For this reason, we have moved from linear filtering to 
nonlinear one, i.e. we do not perform ordinary cross-correlation, but maintain the same basic strategy to look for a sphere-shaped dark region surrounded by lighter tissue. Prior to nonlinear filtering the dataset is smoothed by a 3 by 3 by 3 kernel to reduce the effect of noise. The form of the $3 \mathrm{D}$ kernel we use for the nonlinear filtering is shown in Fig. 2(b). Each voxel in the dataset is checked by doing the following steps:

- The kernel is centered at the current voxel.

- If any voxel in the KERNEL CENTER exceeds the THRESHOLD value, the current voxel is discarded and the next one is checked.

- The number of voxel values under the THRESHOLD in the KERNEL SPHERE and over the THRESHOLD out of the KERNEL SPHERE is now counted. The count is called SUM.

The parameters used in the algorithm are summarized and briefly explained in Table 1. All parameters are capitalized. There are two groups of parameters. Grid-related parameters depend solely on the geometry of the grid of electrodes used, and are further described in Fig. 2(a). Artifact-related parameters depend on the effect metal electrodes cause in the imaging system.

\begin{tabular}{|c||c|c|c|}
\hline \multicolumn{4}{|c|}{ Table 1 - Algorithm Parameters } \\
\hline \hline Type & Parameter & Value & Description \\
\hline \hline \multirow{2}{*}{$\begin{array}{c}\text { Grid } \\
\text { Related } \\
\text { Parameters }\end{array}$} & DISK DIAMETER & $3.8 \mathrm{~mm}$ & Electrode diameter \\
\cline { 2 - 5 } & DISK HEIGHT & $0.5 \mathrm{~mm}$ & Electrode height \\
\cline { 2 - 5 } & OUTER MARGIN & $5.0 \mathrm{~mm}$ & Supporting material margin \\
\hline \hline \multirow{3}{*}{$\begin{array}{c}\text { Artifact } \\
\text { Related }\end{array}$} & KERNEL CENTER & $7.0 \mathrm{~mm}$ & Inner "dark" area \\
\cline { 2 - 4 } Parameters & KERNEL SPHERE & $10.5 \mathrm{~mm}$ & The whole "artifact" \\
\cline { 2 - 4 } & MIN DISTANCE & $7.0 \mathrm{~mm}$ & Minimal electrode distance \\
\cline { 2 - 4 } & MAX DISTANCE & $14.0 \mathrm{~mm}$ & Maximal electrode distance \\
\cline { 2 - 4 } & MIN DIAGONAL & $10.0 \mathrm{~mm}$ & Minimal square diagonal \\
\cline { 2 - 4 } & MAX DIAGONAL & $17.0 \mathrm{~mm}$ & Maximal square diagonal \\
\cline { 2 - 4 } & THRESHOLD & 40 & "Dark" is under threshold \\
\hline
\end{tabular}

Thus, each voxel is either discarded or its position and SUM are recorded. The bigger the sum, more likely the particular voxel is an electrode center. However, there are typically many (thousands) of voxels that were recorded during the nonlinear filtering. At this point the first geometric constraint is introduced. Since electrodes on the grid we use are $10 \mathrm{~mm}$ apart (DISTANCE parameter), then any two estimated electrode centers should not be closer than a certain distance (MIN DISTANCE parameter). By imposing this constraint many of the estimated centers are rejected (the estimated centers that are kept are those with higher SUM).

The output of this step is a set of positions that are likely to be close to positions of possible electrode centers. The estimated positions are guaranteed 


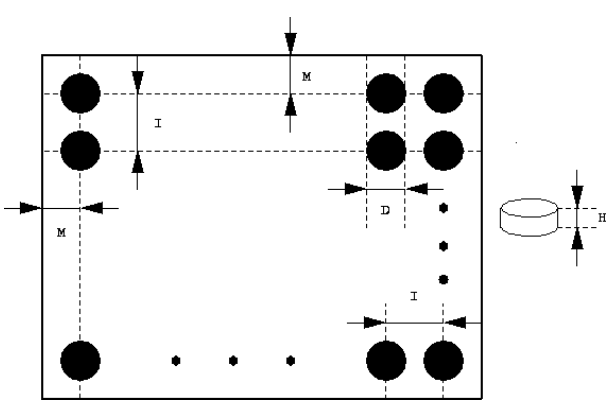

(a)

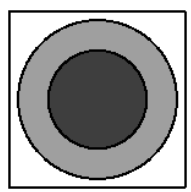

(b)

Fig. 2. Figure (a) shows the grid related parameters, that define its geometry. The black circles represent the electrodes arranged in a grid, while at right a single electrode is show. $\mathrm{M}$ is OUTER MARGIN, D is DISK DIAMETER, $\mathrm{H}$ is DISK HEIGHT and I is DISTANCE. Figure (b) shows a section through the $3 \mathrm{D}$ kernel. The innermost (the darkest) region is kernel center (its diameter is KERNEL CENTER) and the gray ring together with the kernel center is kernel sphere (its diameter is KERNEL SPHERE).

to be at least MIN DISTANCE apart. Typically, a majority of the electrode centers are found, some are missed, and there are some other structures that are wrongly chosen as electrode centers.

\section{$2.2 \quad$ Predictive Filtering}

The purpose of this step is, using the estimated electrode centers, to label some of them, discard the rest and predict the positions of missing ones. The grid patterns are searched from the largest (typically 8 by 8 ) to the smallest ones. Each time a pattern is found, the electrodes belonging to that grid are removed from the list of estimated electrode positions, and then next largest grid pattern is looked for. However, since there are typically a few hundred estimated electrode centers, it would be computationally too expensive to check say an 8 by 8 grid pattern against all possible combinations of estimated electrode centers, including cases with missing electrodes. For this reason we first determine what we call "low level topology". We look for all pairs of estimated centers that might be neighboring electrodes on a grid - these pairs are referred to as "links". Any two estimated centers are at least MIN DISTANCE apart (that was enforced in previous step), and two estimated centers make a link if they are at most MAX DISTANCE apart. Next, we look for all sets of four estimated centers that might compose a square on grid, i.e. that make a 2 by 2 "subgrid". A "square" must have sides composed of already determined "links", and its diagonals must be at least MIN DIAGONAL and at most MAX DIAGONAL. An example of estimated centers and "low level topology" is shown in Fig. 3(b). 


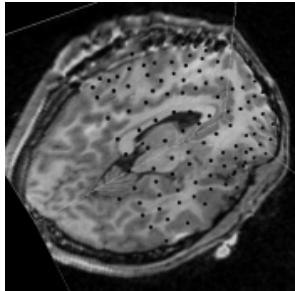

(a)

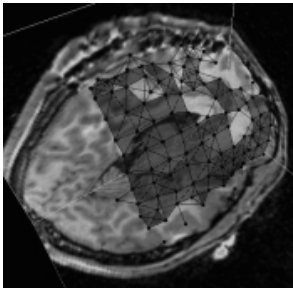

(b)

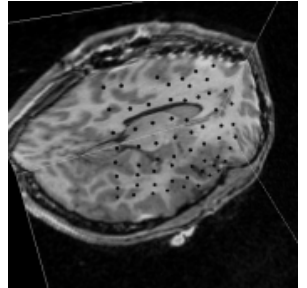

(c)

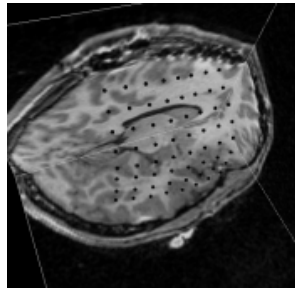

(d)

Fig. 3. Figure (a) shows part of the estimated electrode centers in a post-op MR scan (some of the estimated centers are occluded by the three orthogonal slices through the dataset). One can see a pattern of an 8 by 8 grid, but still the estimated centers are not regularly arranged, some are missing and there are false ones. Figure (b) represents the "low level topology" ("links" and "squares") as defined in the paper. Figure (c) shows the best match for an 8 by 8 grid pattern using the estimated electrode centers and "low level topology", while predicting the missing electrodes. The non-regularity of electrode positions is due to errors in estimated electrode center positions. It is significant in the top row of the grid, since the wires are coming out of the grid at that side causing increased artifacts. Figure (d) shows the electrode positions of the best match after regularization. One can see that the electrode positions form a more regular grid, compared to the one in Figure (c).

Now, having the "low level topology" available, it is much easier to search for grid patterns. For each "square" the pattern is searched for by sliding it over that "square". In other words, a pattern is positioned over the current "square", such that the "square" is in position $(1,1)$ in the pattern, this case is checked, then the pattern is moved such that the "square" is in position $(1,2)$ in the pattern, this case is checked, and so on until the "square" visited all positions in the pattern, both for "vertical" and "horizontal" orientation of the pattern (if the pattern has the same number of rows and columns, then "vertical" and "horizontal" orientations are the same, i.e. only one orientation is checked). For a particular case, say when the current "square" is in position $(2,4)$ in the grid pattern, the nodes in the grid pattern neighboring to the square are predicted using the estimated positions of the four vertices of the "square". Predicted node positions that are close to some estimated centers are replaced by them. Further, the new neighboring nodes in the grid pattern are predicted using the the positions of the "square" vertices and already predicted node positions, and so on until the whole pattern is filled. By doing this, the pattern tries to follow the estimated centers, but if some are missing their positions are predicted. Once this process is finished, it is counted how many node positions, links and squares in the pattern match the estimated electrode positions, "links" and "squares" from the "low level topology". This sum is used as a measure to find the best pattern match. Thus, for each "square" the pattern is moved over all positions 
(and for both orientation), and the best match is determined. An example of the best pattern match is shown in Fig. 3(c).

\subsection{Regularization and Surface Interpolation}

The estimated electrode positions and the predicted positions of the missing electrodes have errors. However, those errors tend to cancel when a surface of a know geometry (representing the grid) is fitted through the best pattern match. The reason why the errors tend to cancel is that there is no preferred direction for error in estimated or predicted electrode centers. We suggest the following way to regularize the grid. If $\boldsymbol{r}_{\mathbf{1}}, \boldsymbol{r}_{\mathbf{2}}, \boldsymbol{r}_{\mathbf{3}}$ and $\boldsymbol{r}_{\mathbf{4}}$ are positions of four electrodes making a square in the best pattern match (the vertices of the square are connected as follows: 1-2-3-4-1), and

$$
\begin{aligned}
& x_{t}=\frac{r_{4}-r_{1}+r_{3}-r_{2}}{\left|r_{4}-r_{1}+r_{3}-r_{2}\right|}, \quad y_{t}=\frac{r_{2}-r_{1}+r_{3}-r_{4}}{\left|r_{2}-r_{1}+r_{3}-r_{4}\right|}, \quad n=x_{t} \times y_{t}, \quad a=\frac{x_{t+y_{t}}}{\left|x_{t}+y_{t}\right|} \\
& \boldsymbol{d}=\frac{\boldsymbol{a} \times \boldsymbol{n}}{|\boldsymbol{a} \times \boldsymbol{n}|}, \quad \hat{\boldsymbol{c}}=\frac{\boldsymbol{a}+\boldsymbol{d}}{|\boldsymbol{a}+\boldsymbol{d}|}, \quad \hat{\boldsymbol{y}}=\frac{\boldsymbol{a}-\boldsymbol{d}}{|\boldsymbol{a}-\boldsymbol{d}|}, \quad \boldsymbol{c}=\frac{\boldsymbol{r}_{\mathbf{1}}+\boldsymbol{r}_{\mathbf{2}}+\boldsymbol{r}_{\mathbf{3}}+\boldsymbol{r}_{\mathbf{4}}}{4},
\end{aligned}
$$

then it is not difficult to see that $\boldsymbol{c}$ is the origin, and $\hat{\boldsymbol{x}}$ and $\hat{\boldsymbol{y}}$ are orthogonal unit vectors of a planar coordinate system fitted through the four vertices and centered at the square center. Furthermore, $\hat{\boldsymbol{x}}$ is approximately in direction 2-3 (and 1-4), while $\hat{\boldsymbol{y}}$ is approximately in the direction 1-2 (and 4-3). This is used to improve the vertex positions, knowing the inter-electrode distance (DISTANCE), as follows:

$$
\left[\begin{array}{l}
\boldsymbol{r}_{\mathbf{1}} \\
\boldsymbol{r}_{\mathbf{2}} \\
\boldsymbol{r}_{\mathbf{3}} \\
\boldsymbol{r}_{\mathbf{4}}
\end{array}\right]_{\text {new }}=\boldsymbol{c}\left[\begin{array}{l}
1 \\
1 \\
1 \\
1
\end{array}\right]+\frac{\text { DISTANCE }}{2}\left[\begin{array}{rr}
-1 & -1 \\
-1 & 1 \\
1 & 1 \\
1 & -1
\end{array}\right]\left[\begin{array}{l}
\hat{\boldsymbol{x}} \\
\hat{\boldsymbol{y}}
\end{array}\right]
$$

This computation is done for each square in the grid. Since a vertex may be shared by two or four squares, its new position is computed as the average of its new positions in each of the squares it belongs to. Let $\mathcal{R}$ be a vector of all the electrode positions in the grid pattern. Combining previous equations for all the "squares" in the grid pattern, one can relate the electrode positions for two subsequent iterations, as $\boldsymbol{\mathcal { R }}_{k+1}=f\left(\boldsymbol{\mathcal { R }}_{k}\right)$, where $k$ is the iteration index. The entire process is iteratively repeated until the electrodes assume steady state positions, i.e. until $\left\|\boldsymbol{R}_{k+1}-\mathcal{R}_{k}\right\|_{\infty}<\epsilon{ }^{4}$. The procedure converges in practice. By doing this, the grid keeps its shape, while enforcing the distances between the electrodes to become closer and closer to the ideal inter-electrode distance. An example of grid regularization is shown in Fig. 3(d).

Regular grids can now easily be interpolated by spline or some other smooth surfaces. However, since the grids are just slightly curved, each square is approximately flat, and practically there is no need to do better than a linear piece-wise

\footnotetext{
${ }^{4}$ Infinity norm of a vector is effectively its maximum element
} 
interpolation. Due to the aforementioned reasons, this interpolation provides almost a smooth surface and very closely satisfies (1). In addition, the surface can be extrapolated to model the grid-supporting material margins, and the surface normals can be computed to properly orient the disk-shaped electrodes. Two examples are shown in Fig. 4.
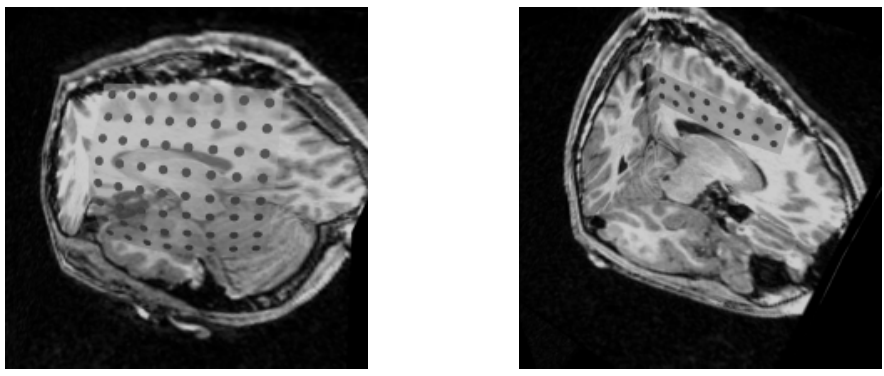

Fig. 4. These two figures show examples of the final electrode grid, represented as a smooth surface with disk-shaped electrodes properly oriented. The electrodes can be colored to denote certain functional areas.

\section{Discussion}

We tried to describe the algorithm in details, so that anyone interested can reproduce it. We have compared the algorithm output to the manually positioned electrodes, concluding that the error is within $1.5 \mathrm{~mm}$, for most of the electrodes within $1 \mathrm{~mm}$ which is sufficient for clinical applications. Our plan for future is to perform extensive validation study of the method, and if necessary to improve it. The whole procedure takes a few minutes on an SGI Octane machine. The method, as currently implemented, works for rectangular grids of electrodes, but can easily be adjusted to work for non-rectangular grids and strips of electrodes as well.

This method is a big step forward in visualization of the electrodes, especially when it is compared to what was used before: either manual clicking on the electrodes, or just mere looking at the slices of post-op MR scans. It is now in clinical use within the Department of Neurosurgery, Yale University.

\section{Acknowledgments}

We are thankful to Dr.Dennis Spencer and Kevin McCarthy from the Department of Neurosurgery, Yale University, for useful discussions and for providing us with data. 


\section{References}

1. A. Chabrerie, F. Ozlen, S. Nakajima, M. Leventon, H. Atsumi, E. Grimson, E. Keeve, S. Helmers, J. Riviello Jr., G. Holmes, F. Duffy, F. Jolesz, R. Kikinis, P. Black: Three-dimensional Reconstruction and Surgical Navigation in Pediatric Epilepsy Surgery. MICCAI'99, Proceedings, (1998) 74-83 991

2. Škrinjar, O., Duncan, J.: Automatic Electrode Grid Extraction from Distorted Post-Op MR Scans. ISMRM'99, Proceedings (1999) 991

3. M. P. do Carmo, Differential Geometry of Curves and Surfaces. Englewood Cliffs, N.J., Prentice-Hall (1976) 